\title{
NOTA SOBRE LA POESÍA ALGORÍTMICA DE RAMÓN LLULL
}

\author{
Carlos Lorenzo Lizalde \\ Universidad de Zaragoza
}

Deus, ab joy xant. Per vos, F.G. discorrens,/ afirmant e negant dubitar destruens,/ concordant $\mathrm{E}$ e I e la $\mathrm{N}$ subponens,/ perlonguant me de $\mathrm{R}$ e de sos conseguens,/ de majoritat fi fas tal acordamens/ que menor e contrari ne sien odiens,/ desirant egualtat myjà comensamens/ en lausar e servir vos, rey omnipotens./ De vos e de lo vostro vul far demostramens/ «propter quid»e «quia» e equiperalmens/ ab majoritat fi per tot lo mesclamens/ de totas les figuras artificialmens:/ si no pusc sens miyà $a b$ miyà $o$ comens:/ d actu d estar obrar vul esser conexens:/ en la obra de dins està y principalmens/ mayoritat e fi, e l contrarixamens/ en la obra de fores, on pauc son inquirens;/ sensual espirital no an egualamens:/ different major es quan es $\mathrm{d}$ acordamens/ que no es de contraris, car pres son aniens./

Si vols far questiò, en T blava comensa;/ tot T mit en los angles e auràs ne siensa:/ a soure questió veges de qual semensa/ venon comensamens concordans senes tensa// e les posicions ages en sovinensa:/ segons elas conclou, car dar $t$ an conexensa./ Duniversal a part sabies far avinensa:/ termens de les figures en la questiò pensa:/ $s i$ as questiò d'A en sos termens comensa,/ e ayso metex fay dels altres a presensa,/mesclant la J en 1 altre, esgardant la valensa/ en tal acordament, que majoritat vensa;/ car major be e gran e ver an convinensa/ de destruir menor, mal, pauc e defalensa:/ si quis plus senblant d'A, ajes en suvinensa:/ subjet e accident no son $d$ una valensa:/ major es $E$ que I, I que $\mathrm{N}$ sens falensa:/ so cové esser ver $\mathrm{d}$ on $\mathrm{E} I$ an crexensa/ en lausar e servir A d on an influensa:/ metàforas faràs, car saber hi comensa.

Comensament miyà e egual, so malbir/que ab majoritat fi $n$ cové concluir/d actu primer segon; car no y poyray falir/ en reyalitat rasò si ò say persegir// Enfre causa e s efectu major fi vul tenir:/ effectu e menor so solen avenir:/ majoritat e Deu, hom no y pot contradir./ Tot quant Deus à en si es egal, a ver dir// es cové mils en luy que $n$ altre, sens falir;/ e tot so es en Deu on mays be s pot segir/ dintre ses dignitatzs, on res no pot falir:/ mils se cové en si que en sos son complir./Si de particular vols lo ver conclusir// dejús universal lo sabies costuir:/ afermar major es que negar, e consir/ que can negar mays es, E ò fay persegir./ Declinaciò fay, car no y poràs falir:/ de materia e forma e de lur 
influir/ corporal spirital ensemblans conjunyir/ los fay tant con poyràs, e no vules mentir./

Qui per Vj mous est'Art discurre la sabia,/ en movent S la T tost saber la poria;/ e si en concloír d inpossíbol volia/ usar pus volenter, molt mils se $n$ aydaria; / que del sobjet prop usàs mays tota via:/ si vuy non potz xausir xauxix en altre dia./ No t vules desputar ab hom de vilania:/ en los ditz que t diran, prin senyal, tota via:/ ab la T prova 1 test, car dar $t$ à maestria./ Primas entencions e segonas volria,/ e ab majoritat fi ne fees conpanya:/ si questiò es d'A o de quel res que sia,/ concorda gran major, falir no y poyràs mia:/ primera 'ntenciò e segona hi sia:/ majoritat e fi obsolulament lia/ totas vetz $a b$ bontat, e que mal no y estia./ Contingent necessari no son d una paria:/ enfre ens e no ens esgarda meloria,/ e máteria e forma en compost pijuria./

Afermant o negant sia 1 test conservatz:/ afermar se cové ab esser, so sabiatz,/ e negar e no esser se son aconpaynatz./ Si t fayl responciò sia Deus reclamatz,/ que per oraciò él te mostre vertatz./Si no potz concluir ab possibilitatz/ en major entitat, inpossibilitatz/ sia $\mathrm{n}$ ton concluir, e auràs ne vertatz./ $\mathrm{D}$ ymaginaciò te garda a totz latz,/ que n ver spiritual no $t$ aja enpatxatz:/ contradictiò es en nos major assatz/ que $n$ Deu, car major es que nos; e es vertatz/ que nos mils conexem Deu mambrant volentatz/ entendre, que ab be, gran, poder, ternitatz; / car ab so d on mays em jugjam, mas va totz latz,/ car Deus es recolibles e entès e amatz,/ e n mays per mays devem d él dire la vertatz./ La figura Deus creet, no s pot mudar, sabiatz,/ $\mathrm{ca}(\mathrm{r})$ sos sabers poders hi sirien mermatz;/e so que sabs per nom serc' ab propietatz./

Ramon LLULL. Regles introductories a la pràctica de la Art Demostrativa. Obres de Ramon Lull. Comissió Editora Lulliana. Mallorca, 1906.

Dios, con gozo canto. Por vos, F.G. discurriendo,/ afirmando y negando el dudar destruyendo,/ concordando $\mathrm{E}$ e I y la $\mathrm{N}$ suponiendo,/prolongándome con $\mathrm{R}$ y con sus consiguientes, / de grandeza y fin haz tales acuerdos/ que menor y contrario no sean contrapuestos,/ deseando igualdad media comenzamos/alabándoos y sirviéndoos, rey omnipotente./ De vos y de lo vuestro quiero hacer demostración/ «propter quid» y «quia» y por equiparación/ con la grandeza y el fin por toda combinación/ de todas las figuras artificialmente:/ si no puedo sin medio, con medio lo comienzo:/ del acto de ser y del obrar quiero ser cognoscente:/ en la obra de dentro ahí está principalmente/ grandeza y fin, y las contradicciones/ en la obra de fuera, donde pocos la buscan;/ sensual y espiritual no tienen adecuación/ diferencia mayor es cuando se trata de concordancia/ (cuando) no son contrarios, pues son más convenientes./

Si quieres preguntar, en $\mathrm{T}$ azul comienza;/ pon todo $\mathrm{T}$ en los ángulos y tendrás la respuesta:/al resolver la cuestión cuida de qué origen/vienen los comienzos concordantes sin tensión,/ y las posiciones ten en cuenta:/ según ellas concluye, pues te han de dar sapiencia./ De universal a particular sabe hacer avenencia:/ de los términos de las figuras en la cuestión piensa:/ si preguntas por $\mathrm{A}$, en sus términos comienza,/ y eso mismo haz de los otros en presencia,/ mezclando la J (la una) en la otra, cuidando la valencia:/ en tal acordamiento, que la grandeza venza;/ pues mayor bien, y grandeza y verdad han conveniencia/ en destruir lo menor, lo malo, lo poco y la falacia:/ si alguien es más semejante de $\mathrm{A}$, ten en cuenta:/ (que) sujeto y accidente no son de igual valencia:/ mayor es E que I, I que $\mathrm{N}$ sin duda:/ conviene sea verdadero donde $\mathrm{E}$ I tienen 
crescencia/ alabando y sirviendo a A de dónde se influencian:/ metáforas harás, pues saber aquí empieza./

Comienzo, media e igual, a su albur/que con grandeza y fin conviene concluir/del acto primero al segundo; no podría pues fallar/en realidad razón si lo sabes perseguir./Entre la causa y su efecto grandeza y fin quiero tener:/ el efecto y la menor se suelen avenir:/ grandeza y Dios, no se pueden contradecir./ Todo cuanto Dios tiene en sí es igual, en buen decir,/ le conviene mejor a él que a otro, sin dudar;/ y todo eso es en Dios donde mejor se puede seguir/ de entre sus dignidades, donde nada puede faltar:/ mejor conviene a él que a lo suyo, su cumplir//Si de particular quieres verdad concluir// de juicio universal sábelo construir:/ afirmar es mayor que negar, y considera/ que no negar es más, Y haylo que perseguir./ Haz la declinación, pues no podrás fallar:/ de materia y forma y de su influencia/ corporal y espiritual hazlos conjugar/ tanto como puedas, y no quieras mentir./

Quien por VI modos este Arte discurre sabiduría/moviendo S, la T todos saber podrían/ y si concluir de imposible quería/ usar más voluntad, mucho más le ayudaría;/ y que del sujeto propio usase más todavía/ si hoy no puedes escoger, elige en otro día./No quieras disputar con hombre de villanía:/ de los dichos que te digan, toma nota, cada vez/ recuerda el Arte mejor que ayudar te podría:/ Con la $T$ prueba el test, pues ha de darte maestría./ Primeras intenciones y segundas querría/ y con grandeza y fin hagas compañía:/ si cuestión es de A o de cualquier cosa que sea,/ concuerda grande y mayor, fallar no podrás la medida:/ primera intención y segunda allí sean:/ la grandeza y el fin absolutamente liga/ cada vez con bondad, y que el mal allí no sea./ Contingente y necesario no son de una paridad:/ entre ente y no ente queda con lo mejor,/ y materia y forma en compuesto peoría./

Afirmando o negando sea el test conservado:/ afirmar se conviene con ser, eso (lo) sabías,/ y negar y no ser se han acompañado./ Si te falta respuesta sea Dios reclamado,/ que por la oración él te muestre la verdad./ Si no puedes concluir con posibilidad/ en la mayor entidad, imposibilidad/sea tu concluir, y tendrás la verdad./ De la imaginación guárdate de todos lados,/ que el ver espiritual no te haya empachado:/ la contradicción es en nos mucho mayor/que en Dios, pues es mayor que nos; $y$ es verdad/ que nosotros mejor conocemos a Dios recordando voluntad/ de entender, que con el bien, lo grande, el poder, la eternidad;/ pues Dios es reconocible y entendido y amado,/ y cada vez más debemos decir de él la verdad./ La figura creó Dios, no se puede cambiar, sabedlo,/ pues sus saberes y poderes serían así mermados;/y eso que sabes por nombre busca con propiedad.

\section{Traducción de Carlos Lorenzo}

Ramón Lull o Llull; castellanizado Raimundo Lulio (1233-1315), nacido en Palma de Mallorca, fue el primer filósofo del ámbito cristiano en Europa, que se expresó en lengua romance, en una habla popular, como muestran estos versos escritos en mallorquín o catalán del siglo XIII; lengua que sin duda entendía el pueblo llano habitante en el territorio de la Corona de Aragón. 
De los mismos existe, la correspondiente versión latína (Manuscrito vaticano y edición de Maguncia -1971- de las Beati Raimundo Lulli Opera Omnia).

La versión original catalana, (catalano-provenzal) que aquí comentamos es un claro exponente de la vocación de universalidad y de difusión de su pensamiento que Llull buscaba, y que él mismo expresa en la introducción al Libro del ascenso y descenso del entendimiento, dirigido a los «hombres seglares» que no han podido recibir una educación (se sobreentiende eclesiástica, en latín canónico).

El poema está dedicado al infante Don Jaime, hijo de Jaime I «el Conquistador», de quien fue preceptor; y fechado en Montpellier, dominio entonces del rey.

Hay que decir, que la difusión de la verdad de Llull se propone es de orden religioso, de acción misional. La orden franciscana, de reciente creación, tendrá en él un esforzado fundador de centros monásticos y de una escuela de traductores de lenguas orientales.

Durante nueve años se dedicó al estudio del árabe, para llevar a las comunidades islámicas la verdad revelada -pero a la vez, razonada, deducida lógicamente; lo que justifica su inclusión en la $\mathrm{H}^{\mathrm{a}}$ de la Filosofía- del Dios uno y trino.

(A causa de lo cual, parece ser que fue lapidado en uno de sus viajes a Bujía, en Túnez; de cuya consecuencia moriría).

Era este dogma trinitario, la marca definitoria de la Iglesia católica respecto del Islam (la cual, como se recordó en el reciente Congreso de Filósofos Jóvenes celebrado en Oviedo, con el lema «Filosofía y Dios»; consideraba por su parte, una herejía, la creencia cristiana en «tres dioses», frente a la pureza monoteísta de la fé en Alá).

Recordemos también, de pasada, que dos siglos y medio más tarde, la extrañeza de otro insigne filósofo aragonés, Miguel Servet, por la expulsión de los árabes, con la supuesta excusa de no acatar este dogma, que Servet no encontró reseñado en la Biblia; le llevó a la hoguera calvinista -que escogió, se puede decir, para no ser quemado por la Inquisición zạragozana, en su propia tierra-.

Pues bien, para no apartarnos del tema, es claro que Ramón Llull es uno de los grandes autores que tendieron un puente entre el pensamiento de Oriente y el de Occidente. Llull pretendía, en principio, unificar las tres religiones.

En la intuición lógica luliana tuvo mucho que ver la cultura arábigo-hispánica, islámica y judía; la cual, no lo olvidemos, aparte de transmitir, conservar y perfeccionar el legado de la cultura clásica griega y especialmente de Platón y Ariștóteles; supo desarrollar como ninguna en esos siglos medievales, varias ramas de la ciencia, como la medicina (posible influencia en Servet), la matemática, la cartografía, la astronomía o la alquimia.

No por casualidad, dos siglos antes, el reino taifa de Zaragoza había dado, con figuras de la talla de Avempace, la primera escuela filosófica medieval (1020-1118), (como señala Joaquín Lomba en Filosofía islámica en Zaragoza., Zaragoza: Diputación General de Aragón, 1987).

Para Julián Ribera y así mismo para Asín Palacios (en «Mohidín», Homenaje a Menéndez Pelayo. Madrid: Librería V. Suárez, 1911) hay una influencia directa en Llull de Ibn Arabí de 
Murcia (1165-1240), en especial por las formas simbólicas expositivas de su ciencia, unidas a la mística intuitiva, antiaverroísta, de ambos.

Roger Arnaldez, en su capítulo de la $\mathrm{H}^{\mathrm{a}}$ Gral. de las Ciencias (Paris: PUF, 1960; Barcelona: Destino, 1973. dirigida por R. Taton), dedicado a la ciencia árabe, llega aver en el Ars magna luliana, una imitación de la zairja o «máquina para pensar los acontecimientos», construida por los astrólogos árabes.

Y Luis Racionero, en Raimon o el seny fantàstic (Barcelona: Laia, 1985); relaciona el «Ars» y otras obras lulianas, con el misticismo sufí y con la cábala judía. Elementos éstos, presentes en la cultura de Aragón, desde los tiempos de la mencionada Escuela zaragozana de filosofía. La propia Mallorca había dado pensadores como Abu Bakr, discípulo del gran maestro del sufismo Ibn al-Arif (vd. J. Lomba, op. cit.).

En cualquier caso, es el cruce de culturas que se dan cita en la Península y su ámbito de dominio en estos siglos del Medievo, el que da lugar a esta «iluminación» luliana que es el «Ars magna»; verdadero avance del cálculo lógico posterior.

«Metageometría metafísica» que aunque de un «simbolismo rudimentario», constituye no obstante un precedente del «cálculo automático» de los actuales «ordenadores y computadoras», como nos dice el Profesor Cruz Hernández (El pensamiento de Ramón Llull, Madrid: Castalia, 1977).

Miquel Batllori ha destacado su influencia en el pensamiento renacentista europeo, en autores como L.Valla, M.Ficino o G.Pico della Mirandola. Influencia que se prolonga en el Barroco.

El propio Leibniz reconoce en el Arte combinatoria, un antecedente de su cálculo infinitesimal.

El interés por la lógica luliana no ha cesado nunca, y un filósofo de la ciencia contemporáneo tan reputado como Martin Gardner («The Ars Magna of Ramon Llull», en Logic Machines and Diagrams. Nueva York: McGraw-Hill, 1958.), lo muestra.

La forma poética de este escrito, obedece, por una parte a un afán didáctico y mnemotécnico. La insistencia y repetición de algunas reglas bastarían para reconocer este aspecto.

Más que un poema, sería mas bien una forma de oración -la cual ha utilizado habitualmente este mismo recurso a la rima y al ritmo-.

No sería tanto un poema, en sentido literario o lírico, en el que la intención estética es primordial; sino poesía, en su sentido originario de poiesis, de creación de un artefacto discursivo aclarador o mostrador de una verdad, y, en este caso, además indicador de un método, de unas reglas prácticas de aplicación, de construcción de un cálculo.

Aunque no olvidemos que Ramón Llull ocupa un lugar destacado en la literatura en catalán; aspecto éste, mucho más estudiado contemporáneamente que el filosófico.

El joven Llull es un cortesano del rey Jaime de Aragón. Como tal, cultiva el amor cortés, el «arte de amar» de los trovadores provenzales.

Trovas, que serán luego, tras su conversión, místicas, de amor a Dios, como en el libro De amigo y de amado. 
Como Agustín de Hipona, también este caballero será un día iluminado, verá la luz de la verdad tan clara, que abandonará todo para comunicarla. Con tal ardor, que visitará al papa Juan XXI -Pedro Hispano-, maestro en lógica de raigambre aviceniana- proponiéndole una cruzada.

Tanto uno como otro combatirán el «averroísmo latino» parisino: no hay doble verdad. Una es la naturaleza, como reflejo divino, y una la razón humana, manifestación asimismo de Dios.

Como en Hegel, todo lo real es racional, pues procede de Dios, por eso es perfectamente demostrable. Deducible según un orden que es el mismo orden de los seres creados.

Siguiendo a Antonio Alegre en su clara y sintética presentación del Libro del ascenso y descenso del entendimiento, recordamos aquí, para la interpretación de las Reglas, que para Llull «la escala de los seres se autodespliega a causa de los atributos (o dignidades) de Dios». Estos son, tal como aparecen en el poema y luego distribuidos en las «ruletas» combinatorias formadas por triángulos, los siguientes: Bondad, Grandeza, Eternidad, Poder, Sabiduría, Voluntad, Virtud, Verdad, Gloria, Diferencia, Concordancia, Principio, Medio, Fin, Igualdad.

Luis Racionero (op. cit.) nos muestra la similitud notoria con los sefirots de la Cábala: Gloria, Sabiduría, Verdad; Bondad, Potestad, Virtud; Duración, Esplendor, Fundamento.

Para Llull, -siguiendo a A.Alegre-, «las esencias particulares lo son en cuanto participaciones finitas de las criaturas en estos atributos». «Estas ideas o arquetipos ofician como modelos ejemplares. Entender el mundo es comprender, ver en él las ideas universales; comprender los atributos divinos consiste en verlos ejemplificados en el mundo».

«A partir de los axiomas, evidentes, fondo de toda razón, combinados con razonamientos, mediante los cuales comprendemos las relaciones necesarias entre las cosas... Lulio trataba de probar, mecánicamente, cómo el juego relacional de los individuos y del mundo no es otra cosa que reflejo e implicación en el juego real y relacional de los atributos divinos».

Antes de comentar el poema, hay que mostrar su puesta en práctica, a través de lo que Llull llama las figuras.

La figura de $\mathrm{T}$, se divide en dos. La primera es circular, compuesta de 5 triángulos de diversos colores: azul, verde, amarillo, rojo y negro.

La segunda fugura consta de 120 cámaras, obtenidas a base de combinar, de dos en dos, los elementos de la primera figura $\left(15^{*} 15+15 / 2=120\right)$.

Comienza el poema o cántico, como el propio autor lo califica, alabando a Dios; y así mismo acaba la estrofa tras el primer punto. La intención es, pues, manifiesta; el artificio que sigue ha de mostrar la mayor gloria de Dios.

A través de él y de sus atributos, convenientemente simbolizados y estratégicamente situados en las figuras triangulares, podemos ir discurriendo, esto es, encontrando juicios afirmativos y negativos, que destruyan toda duda. Este discurrir tiene el doble sentido de pensar, de razonar (discurso racional), y de seguir el recorrido combinatorio de los símbolos literales, mecánicamente: «por vos, F (concordancia) y G (contrariedad) discurriendo», esto es, recorriendo. «concordando E (diferencia) e I (medio) y la N (menoridad) suponiendo», «De 
grandeza y de fin haz tales acuerdos»; esto es, partiendo del atributo o manifestación mayor de Dios, de arriba hacia abajo, llegar hasta el fin o realización de cada ser, de cada entidad, de manera demostrativa. Cuidando que menor $(\mathrm{N})$, y contrario $(\mathrm{G})$ no se contrapongan; para lo que hay que buscar antes bien la igualdad (M).

A continuación explicita otra vez, cómo pretende, con este Arte, «hacer demostración» de Dios y de lo suyo. Siendo lo suyo el mundo, la naturaleza, los seres, incluido el ser humano y su razón.

Puede verse aquí una concepción de Dios neoplatónica o plotiniana, de la emanación del ser supremo, y su «descenso» por todos los niveles del ser creado. Que muestra la influencia de la mística de Ibn Arabí.

De manera que cualquier practicante del «arte» puede así conocer la actualización de los seres por medio de la acción de Dios a través de la propia teleología natural. Y ese obrar; esa acción, se corresponde con la operación combinatoria de los símbolos representando las esencias: «la obra de dentro» y «la obra de fuera». Lo intrínseco y lo extrínseco de la escala de los seres, se corresponde con las cámaras halladas por las combinaciones de las figuras; así como por las combinaciones desechadas por estar «mal construidas» según las reglas del cálculo: «las contradicciones (están) en la obra de fuera».

«Sensual y espiritual no tienen igualamiento». Esta frase nos muestra el dualismo luliano, de raigambre platónico-agustiniana.

Aunque más que dualismo, se da aquí una escala de los seres, con múltiples escalones. Lo sensual responde a lo que podemos percibir por los sentidos: vista, oído, olfato, gusto, tacto y «afato». Lo espiritual corresponde a Dios, al ángel y al alma racional.

Cuando se trata de hacer concordar estos atributos simbolizados en la ruleta, hay que tener un cuidado especial, para ver si tienen una verdadera correspondencia en los seres reales. (Pongamos por ejemplo, siguiendo a LLull, las combinaciones en E, siguientes: intelectual e intelectual, sensual e intelectual, sensual y sensual. Poniéndolas a prueba mecánicamente hasta su fin, para ver si son posibles o imposibles, entes o no entes.)

La segunda estrofa es técnica; explica al discípulo, al practicante del Arte, cómo debe plantear una pregunta: empezando por el triángulo azul (que consta de Dios -B-, Criatura C- y Operación -D-) y superponiendo el resto de los triángulos, para establecer todas las combinaciones. Cuidando primero de respetar el orden de las mismas (empezando por el principio -H-), y luego, eliminando las que no sean concordantes.

La regla técnica de ir haciendo concordancias en descenso, «de universal a particular», se corresponde, naturalmente con la escala real de los seres. En estas combinaciones ha de «vencer» siempre lo mayor sobre lo menor, lo positivo sobre lo negativo, el bien sobre el mal, el ser sobre el no-ser. Si se tiene cuidado en aplicar bien las reglas, la verdad será siempre evidente y triunfará sobre la falsedad.

La falsedad no sería pues, sino el error; la aplicación errónea de las reglas de formación de conceptos. Para ello, recomendación al discípulo de guardar «la valencia» (es como si ajustáramos, sobre el papel, una reacción química), o si se quiere «el valor» de verdad (de la lógica proposicional, por ejemplo). 
«Mayor es $\mathrm{E}$ (diferencia) que I (medio), I que $\mathrm{N}$ (menoridad)... conviene que sea cierto, cuando E I -diferencia y medio- van ascendiendo». Reglas éstas que se resumen otra vez al acabar la estrofa, en la premisa de que el orden de lo real, de lo divino, se corresponde con el orden de la lógica racional, del conocimiento. Este es cognoscible porque recibe su influencia de Dios, quien, consecuentemente con esta equiparación es aquí alabado como «A».

«Metáforas harás», combinaciones, conclusiones, en las que la razón, que nos muestra a la vez el orden de la realidad, no puede fallar; si se aplica, si se «prosigue» bien el método.

Y a continuación, ejemplos de esa afirmación. Por un lado se «avienen» Dios con su atributo la grandeza y con la modalidad lógica y ontológica de la causa; mientras que por el otro extremo, la menoridad se «aviene» con el efecto y el fin.

La totalidad de las cosas, nos dice en los versos siguientes, se articula según principios que son los atributos de Dios: «e tot so es en Deu on mays be s pot segir/ dintre ses dignitatz, on res no pot falir».

Los siguientes versos dan las reglas axiomáticas de que lo universal y la afirmación son superiores a lo particular y a la negación.

La construcción del juicio no puede fallar si se mantiene esa regla y se declina o deriva de arriba abajo, de las esencias universales que son los atributos divinos a su ejemplificación en el mundo.

Otra regla como las anteriores es la de conocer la similitud entre el cuerpo y la materia por un lado, y el espíritu y la forma por otro: las causas aristotélicas son principios de conocimiento, e isomorfas con la realidad.

Este Arte, por el que discurre la sabiduría, esto es el entender el mundo de las criaturas como despliegue jerarquizado de las dignidades arquetípicas de Dios; todos lo pueden manejar, cualquier ser humano que tenga un poco de cuidadao en mover bien las ruletas.

Hay que precaverse también de otros problemas, como son el estado anímico del practicante del arte: «si hoy no puedes decidir» déjalo, ya lo harás otro día. Además hay que poner voluntad en encontrar las conclusiones buscadas.

Y no dejarse engañar por supuestas lógicas villanas o malintencionadas. Todo lo más «escucha los dichos que te digan», para ponerlos a prueba con el Arte combinatoria.

Una vez más insiste Llull en recordar las jerarquías, las buenas y malas conjunciones: de la Bondad y la Grandeza a través de la primera y segunda intenciones; para derivarlas y encontrarlas otra vez en el fin.

Hasta adquirir maestría en el arte de la demostración por combinaciones mecánicas, hay sobre todo que practicar.

Pero, en definitiva, nos dice en la última estrofa; el garante de que el Arte funcione, es Dios. Garante de la lógica y de la verdad. Porque él es la verdad, y por eso hemos de proclamarla por todas partes, entre quienes no lo tienen claro, porque no han sido iluminados.

Ultimas recomendaciones al practicante de este arte demostrativo de la gloria de Dios: No te preocupes si a veces no acabas de ver claro, si caes en contradicción, pues es normal dada nuestra pequeñez con respecto a Dios. Ten cuidado también con la imaginación, con 
despistarte de las reglas. Y que la contemplación de la verdad -o la práctica del Arte-no te empachen o te ofusquen por exceso de claridad. Si te falla la respuesta, reza y «sea Dios reclamado». El te mostrará la verdad, ya que «la figura creó Dios». Dios informó, dió la forma a los seres del mundo. Por ello podemos reconocerlo, conocer a través de sus criaturas, como sus emanaciones: «el bien, la grandeza, el poder, la eternidad».

$\mathrm{Y}$ así le amaremos no sólo emotivamente, sino también porque le entendemos, de manera racional.

El poema acaba resumiendo su sentido, para el creyente: y eso que sabías ya por la palabra (por la palabra revelada), búscalo con propiedad, es decir, razónalo. $O$, dicho en lenguaje lógico-matemático, demuestra como teoremas los axiomas de partida. El cálculo, más o menos axiomatizado, sirve aquí a la mística teológica, en una conjunción que atrae efectivamente con ese «eros» en que la «buena obra de arte», la obra bien construida, se confunde con la belleza, y arrastra finalmente a la razón hacia el asentimiento. 